\title{
Certain Class of Generating Functions for the Incomplete Hypergeometric Functions
}

\author{
Junesang Choi ${ }^{1}$ and Praveen Agarwal ${ }^{2}$ \\ ${ }^{1}$ Department of Mathematics, Dongguk University, Gyeongju 780-714, Republic of Korea \\ ${ }^{2}$ Department of Mathematics, Anand International College of Engineering, Jaipur 303012, India
}

Correspondence should be addressed to Junesang Choi; junesang@mail.dongguk.ac.kr

Received 4 June 2014; Accepted 17 July 2014; Published 18 August 2014

Academic Editor: Ali H. Bhrawy

Copyright (c) $2014 \mathrm{~J}$. Choi and P. Agarwal. This is an open access article distributed under the Creative Commons Attribution License, which permits unrestricted use, distribution, and reproduction in any medium, provided the original work is properly cited.

Generating functions play an important role in the investigation of various useful properties of the sequences which they generate. In this paper, we aim to establish certain generating functions for the incomplete hypergeometric functions introduced by Srivastava et al. (2012). All the derived results in this paper are general and can yield a number of (known and new) results in the theory of generating functions.

\section{Introduction and Definitions}

A lot of research work has recently come up on the study and development of the familiar incomplete Gamma type functions like $\gamma(s, x)$ and $\Gamma(s, x)$ given in $((1))$ and $((2))$, respectively. The study of incomplete Gamma functions has a very long history (see, e.g., [1]) and now stands on fairly firm footing through the research contributions of various authors (see, e.g., [2-17]). Incomplete Gamma functions are important special functions and their closely related ones are widely used in physics and engineering; therefore, they are of interest to physicists, engineers, statisticians, and mathematicians.

The theory of the incomplete Gamma functions, as a part of the theory of confluent hypergeometric functions, has received its first systematic exposition by Tricomi [18] in the early 1950s. The familiar incomplete Gamma functions $\gamma(s, x)$ and $\Gamma(s, x)$ are defined, respectively, by

$$
\begin{gathered}
\gamma(s, x):=\int_{0}^{x} t^{s-1} e^{-t} d t, \quad(\Re(s)>0 ; x \geq 0), \\
\Gamma(s, x):=\int_{x}^{\infty} t^{s-1} e^{-t} d t, \quad(x \geq 0 ; \Re(s)>0 \text { when } x=0) .
\end{gathered}
$$

The following decomposition formula holds:

$$
\gamma(s, x)+\Gamma(s, x)=\Gamma(s), \quad(\Re(s)>0),
$$

where $\Gamma(s)$ is the familiar Gamma function defined by

$$
\Gamma(s):=\int_{0}^{\infty} t^{s-1} e^{-t} d t, \quad(\Re(s)>0) .
$$

Historically, ((1)) and ((2)) were first studied in 1877 for $x=1$ by Prym [1]. The functions ((1)) and ((2)) are also referred to as Prym's functions. For general $x>0$ (even for $x<0$ ), the function $((2))$ appears in Exercises de Calcul Integral by Legendre [19] and in some of his later works.

The function $\Gamma(s, x)$ can be expressed in terms of Tricomi's confluent hypergeometric function $\Psi(a, c ; x)$ as follows (see [6, page 266, Equation 6.9.2(21)]):

$$
\Gamma(s, x):=e^{-x} \Psi(1-s, 1-s ; x) .
$$


In terms of the Gamma function $\Gamma(z)$, Pochhammer symbol $(\lambda)_{n}$ is defined (for $\lambda \in \mathbb{C}$ ) by (see, e.g., [10, page 2 and pages $4-6]$ )

$$
\begin{aligned}
(\lambda)_{n} & := \begin{cases}1, & (n=0) \\
\lambda(\lambda+1) \cdots(\lambda+n-1), & (n \in \mathbb{N}:=\{1,2,3, \ldots\})\end{cases} \\
& =\frac{\Gamma(\lambda+n)}{\Gamma(\lambda)}, \quad\left(\lambda \in \mathbb{C} \backslash \mathbb{Z}_{0}^{-}\right),
\end{aligned}
$$

where $\mathbb{C}$ and $\mathbb{Z}_{0}^{-}$denote the sets of complex numbers and nonpositive integers, respectively.

Recently, Srivastava et al. [16] introduced and studied some fundamental properties and characteristics of a family of the following two potentially useful generalized incomplete hypergeometric functions defined as follows:

$$
\begin{array}{r}
{ }_{p} \gamma_{q}\left[\begin{array}{r}
\left.\left(a_{1}, x\right), a_{2}, \ldots, a_{p} ; z\right] \\
b_{1}, \ldots, b_{q} ;
\end{array}\right] \\
:=\sum_{n=0}^{\infty} \frac{\left(a_{1} ; x\right)_{n}\left(a_{2}\right)_{n} \cdots\left(a_{p}\right)_{n}}{\left(b_{1}\right)_{n} \cdots\left(b_{q}\right)_{n}} \cdot \frac{z^{n}}{n !}, \\
{ }_{p} \Gamma_{q}\left[\begin{array}{c}
\left.\left(a_{1}, x\right), a_{2}, \ldots, a_{p} ; z\right] \\
b_{1}, \ldots, b_{q} ;
\end{array}\right] \\
:=\sum_{n=0}^{\infty} \frac{\left[a_{1} ; x\right]_{n}\left(a_{2}\right)_{n} \cdots\left(a_{p}\right)_{n}}{\left(b_{1}\right)_{n} \cdots\left(b_{q}\right)_{n}} \cdot \frac{z^{n}}{n !},
\end{array}
$$

where $\left(a_{1} ; x\right)_{n}$ and $\left[a_{1} ; x\right]_{n}$ are certain interesting generalizations of the Pochhammer symbol $(\lambda)_{n}$ which are defined, in terms of the incomplete Gamma type functions $\gamma(s, x)$ and $\Gamma(s, x)$ given in $((1))$ and $((2))$, by

$$
\begin{aligned}
& (\lambda ; x)_{\nu}:=\frac{\gamma(\lambda+\nu, x)}{\Gamma(\lambda)}, \quad(\lambda, v \in \mathbb{C} ; x \geq 0), \\
& {[\lambda ; x]_{\nu}:=\frac{\Gamma(\lambda+\nu, x)}{\Gamma(\lambda)}, \quad(\lambda, \nu \in \mathbb{C} ; x \geq 0) .}
\end{aligned}
$$

These incomplete Pochhammer symbols $(\lambda ; x)_{v}$ and $[\lambda ; x]_{v}$, which were defined by Srivastava et al. [16], like $((3))$, also satisfy the following decomposition relation:

$$
(\lambda ; x)_{v}+[\lambda ; x]_{v}=(\lambda)_{\nu}, \quad(\lambda, v \in \mathbb{C} ; x \geq 0) .
$$

Remark 1. As already mentioned by Srivastava et al. [16, Remark 7] (see also [17, page 3220, Remark]), since

$$
\begin{gathered}
\left|(\lambda ; x)_{n}\right| \leq\left|(\lambda)_{n}\right|, \\
\left|[\lambda ; x]_{n}\right| \leq\left|(\lambda)_{n}\right|, \\
(n \in \mathbb{N} ; \lambda \in \mathbb{C} ; x \geq 0),
\end{gathered}
$$

the precise (sufficient) conditions under which the infinite series in the definitions $((7))$ and $((8))$ would converge absolutely can be derived from those that are well-documented in the case of the generalized hypergeometric function ${ }_{p} F_{q}$ $(p, q \in \mathbb{N}$ ) (see, for details, [20, pages 72-73] and [11, page 20]; see also [21-23]). Indeed, in their special case when $x=0$, both ${ }_{p} \gamma_{q}(p, q \in \mathbb{N})$ and ${ }_{p} \Gamma_{q}(p, q \in \mathbb{N})$ would reduce immediately to the extensively investigated generalized hypergeometric function ${ }_{p} F_{q}(p, q \in \mathbb{N}$ ) (see, e.g., [20, Chapter 5]; see also [10, Section 1.5]). Furthermore, as an immediate consequence of the definitions $((7))$ and $((8))$, we have the following decomposition formula:

$$
\begin{aligned}
& { }_{p} \gamma_{q}\left[\begin{array}{c}
\left.\left(a_{1}, x\right) a_{2}, \ldots, a_{p} ; z\right] \\
b_{1}, \ldots, b_{q} ;
\end{array}\right] \\
& \quad+{ }_{p} \Gamma_{q}\left[\begin{array}{c}
\left(a_{1}, x\right), a_{2}, \ldots, a_{p} ; z \\
b_{1}, \ldots, b_{q} ;
\end{array}\right] \\
& \quad={ }_{p} F_{q}\left[\begin{array}{l}
a_{1}, \ldots, a_{p} ; \\
b_{1}, \ldots, b_{q} ;
\end{array}\right],
\end{aligned}
$$

in terms of the familiar generalized hypergeometric function ${ }_{p} F_{q}(p, q \in \mathbb{N})$.

Generating functions play an important role in the investigation of various useful properties of the sequences which they generate. They are used in finding certain properties and formulas for numbers and polynomials in a wide variety of research subjects, indeed, in modern combinatorics. For a systematic introduction to, and several interesting (and useful) applications of, the various methods of obtaining linear, bilinear, bilateral, or mixed multilateral generating functions for a fairly wide variety of sequences of special functions (and polynomials) in one, two, and more variables, among much abundant literature, we refer to the extensive works by Srivastava and Manocha [24] and Agarwal and Koul [25]. In this regard, in fact, a remarkably large number of generating functions involving a variety of special functions have been developed by many authors (see, e.g., $[24,26]$; see also [27]). Also many generating functions containing the incomplete hypergeometric functions $((7))$ and $((8))$ have been presented (see, e.g., [17, Corollary 3]). Here, motivated mainly by the works of both Chen and Srivastava [28] and Srivastava and Cho [17], we present certain generating functions involving the incomplete hypergeometric functions $((7))$ and $((8))$. Furthermore, it should be mentioned in passing that our results in the present paper are established by using a different method employed by [17].

\section{Generating Functions for the Incomplete Hypergeometric Functions}

In this section, we establish certain generating functions for the incomplete hypergeometric functions $((7))$ and $((8))$ asserted by Theorem 2 . 
Theorem 2. The following generating functions hold true:

$$
\begin{aligned}
& \sum_{k=0}^{\infty}\left(\begin{array}{c}
\alpha+k-1 \\
k
\end{array}\right){ }_{p} \gamma_{q+1}\left[\begin{array}{c}
\left(a_{1}, x\right), a_{2}, \ldots, a_{p} ; \\
1-\alpha-k, b_{1}, \ldots, b_{q} ;
\end{array}\right] t^{k} \\
& =(1-t)_{p}^{-\alpha} \gamma_{q+1}\left[\begin{array}{c}
\left(a_{1}, x\right), a_{2}, \ldots, a_{p} ; \\
1-\alpha, b_{1}, \ldots, b_{q} ;
\end{array} z(1-t)\right], \\
& (x \geq 0 ; \alpha \in \mathbb{C} ;|t|<1), \\
& \sum_{k=0}^{\infty}\left(\begin{array}{c}
\alpha+k-1 \\
k
\end{array}\right){ }_{p} \Gamma_{q+1}\left[\begin{array}{c}
\left(a_{1}, x\right), a_{2}, \ldots, a_{p} ; \\
1-\alpha-k, b_{1}, \ldots, b_{q} ;
\end{array}\right] t^{k} \\
& =(1-t)^{-\alpha}{ }_{p} \Gamma_{q+1}\left[\begin{array}{c}
\left(a_{1}, x\right), a_{2}, \ldots, a_{p} ; \\
1-\alpha, b_{1}, \ldots, b_{q} ;
\end{array} ;(1-t)\right], \\
& (x \geq 0 ; \alpha \in \mathbb{C} ;|t|<1) .
\end{aligned}
$$

Proof. For convenience, let the left-hand side of ((13)) be denoted by $\mathcal{S}$. Applying the series expression of $((7))$ to $\mathcal{S}$, we get

$$
\begin{aligned}
\mathcal{S}= & \sum_{k=0}^{\infty}\left(\begin{array}{c}
\alpha+k-1 \\
k
\end{array}\right) \\
& \cdot\left(\sum_{n=0}^{\infty} \frac{\left(a_{1} ; x\right)_{n}\left(a_{2}\right)_{n} \cdots\left(a_{p}\right)_{n}}{(1-\alpha-k)_{n}\left(b_{1}\right)_{n} \cdots\left(b_{q}\right)_{n}} \cdot \frac{z^{n}}{n !}\right) t^{k} .
\end{aligned}
$$

Using the following known identities (see, e.g., [10, page 5]):

$$
\begin{aligned}
\left(\begin{array}{l}
\alpha \\
n
\end{array}\right) & =\frac{\Gamma(\alpha+1)}{n ! \Gamma(\alpha-n+1)}, \quad\left(n \in \mathbb{N}_{0} ; \alpha \in \mathbb{C}\right), \\
\frac{\Gamma(\alpha-n)}{\Gamma(\alpha)} & =\frac{(-1)^{n}}{(1-\alpha)_{n}}, \quad\left(n \in \mathbb{N}_{0} ; \alpha \in \mathbb{C} \backslash \mathbb{Z}\right),
\end{aligned}
$$

$\mathbb{Z}$ being the set of integers and $\mathbb{N}_{0}:=\mathbb{N} \cup\{0\}$, we can prove the following identity (see [28, page 169]):

$$
\begin{aligned}
(1-\alpha-k)_{n}= & (1-\alpha)_{n}\left(\begin{array}{c}
\alpha+k-1 \\
k
\end{array}\right) \\
& \cdot\left(\begin{array}{c}
\alpha-n+k-1 \\
k
\end{array}\right)^{-1}, \quad\left(k, n \in \mathbb{N}_{0}\right) .
\end{aligned}
$$

By changing the order of summations in $((15))$ and using the identity ((17)), after little simplification, we have

$$
\begin{aligned}
\mathcal{S}= & \sum_{n=0}^{\infty} \frac{\left(a_{1} ; x\right)_{n}\left(a_{2}\right)_{n} \cdots\left(a_{p}\right)_{n}}{(1-\alpha)_{n},\left(b_{1}\right)_{n} \cdots\left(b_{q}\right)_{n}} \\
& \cdot \frac{z^{n}}{n !} \sum_{k=0}^{\infty}\left(\begin{array}{c}
\alpha-n+k-1 \\
k
\end{array}\right) t^{k} .
\end{aligned}
$$

We find that the inner sum in $((18))$ is the generalized binomial expansion

$$
\sum_{k=0}^{\infty}\left(\begin{array}{c}
\alpha-n+k-1 \\
k
\end{array}\right) t^{k}=(1-t)^{-\alpha+n}, \quad(|t|<1) .
$$

Finally, replacing the inner sum of $((18))$ by the identity $((19))$ yields our desired result ((13)).

It is easy to see that a similar argument as in the proof of ((13)) will establish the result ((14)). This completes the proof of Theorem 2.

Remark 3. Recently, Srivastava and Cho [17] presented a very general class of certain interesting generating functions involving the incomplete hypergeometric functions $((7))$ and $((8))$ by essentially using the following interesting and useful unified expansion formula given by Gould (see [29, page 196, Equation (9)]; see also [17, page 3221]):

$$
\begin{aligned}
& \sum_{k=0}^{\infty} \frac{\kappa}{\kappa+(\beta+1) n}\left(\begin{array}{c}
\alpha+(\beta+1) n \\
n
\end{array}\right) t^{n} \\
& \quad=(1+\xi)^{\alpha} \sum_{n=0}^{\infty}(-1)^{n}\left(\begin{array}{c}
\alpha-\kappa \\
n
\end{array}\right)\left(\begin{array}{c}
n+\frac{\kappa}{\beta+1} \\
n^{-1}
\end{array}\right)\left(\frac{\xi}{1+\xi}\right)^{n},
\end{aligned}
$$

where $\alpha, \beta$, and $\kappa$ are complex numbers independent of $n$ and $\xi$ is a function of $t$ defined implicitly by

$$
\begin{aligned}
& \xi=t(1+\xi)^{\beta+1} \\
& \zeta(0)=0 .
\end{aligned}
$$

The results [17, Corollary 3] look very similar to those given in Theorem 2. Yet, it is easy to see that they cannot be special or general cases of the other one's results.

\section{Further Generalization of the Generating Functions for the Incomplete Hypergeometric Functions}

A further generalization of the incomplete hypergeometric functions $((7))$ and $((8))$ is given in the following definition.

Definition 4. Let us introduce two sequences $\left\{\Lambda_{k}^{(\alpha, \beta)}(z)\right\}_{k=0}^{\infty}$ and $\left\{\Omega_{k}^{(\alpha, \beta)}(z)\right\}_{k=0}^{\infty}$ defined by

$$
\begin{aligned}
\Lambda_{k}^{(\alpha, \beta)}(z)=\Lambda_{k}^{(\alpha, \beta)}\left[\begin{array}{r}
\left(a_{1}, x\right), a_{2}, \ldots, a_{p} ; z \\
b_{1}, \ldots, b_{q} ;
\end{array}\right] \\
={ }_{p} \gamma_{q+\beta}\left[\begin{array}{r}
\left(a_{1}, x\right), a_{2}, \ldots, a_{p} ; z \\
\Delta(\beta ; 1-\alpha-k), b_{1}, \ldots, b_{q} ;
\end{array}\right], \\
(x \geq 0 ; \beta \in \mathbb{N} ; \alpha \in \mathbb{C}), \\
\Omega_{k}^{(\alpha, \beta)}(z)=\Omega_{k}^{(\alpha, \beta)}\left[\begin{array}{r}
\left.\left(a_{1}, x\right), a_{2}, \ldots, a_{p} ; z\right] \\
b_{1}, \ldots, b_{q} ;
\end{array}\right] \\
\left.\left(a_{1}, x\right), a_{2}, \ldots, a_{p} ; z\right], \\
x(x \geq 0 ; \beta \in \mathbb{N} ; \alpha \in \mathbb{C}),
\end{aligned}
$$


where, for convenience, $\Delta(\beta ; \alpha)$ abbreviates the array of $N$ parameters as follows:

$$
\frac{\alpha}{\beta}, \frac{\alpha+1}{\beta}, \ldots, \frac{\alpha+\beta-1}{\beta}, \quad(\alpha \in \mathbb{C} ; \beta \in \mathbb{N}) .
$$

Then, as in Theorem 2, we can give the following generating functions for the generalized incomplete hypergeometric functions ((22)) asserted by Theorem 5 .

Theorem 5. Each of the following identities holds true:

$$
\begin{gathered}
\sum_{k=0}^{\infty}\left(\begin{array}{c}
\alpha+m+k-1 \\
k
\end{array}\right) \Lambda_{m+k}^{(\alpha, \beta)}(z) t^{k} \\
=(1-t)^{-\alpha-m} \Lambda_{m}^{(\alpha, \beta)}\left(z(1-t)^{\beta}\right), \\
\left(x \geq 0 ; m \in \mathbb{N}_{0} ; \alpha \in \mathbb{C} ; \beta \in \mathbb{N} ;|t|<1\right), \\
\sum_{k=0}^{\infty}\left(\begin{array}{c}
\alpha+m+k-1 \\
k
\end{array}\right) \Omega_{m+k}^{(\alpha, \beta)}(z) t^{k} \\
=(1-t)^{-\alpha-m} \Omega_{m}^{(\alpha, \beta)}\left(z(1-t)^{\beta}\right),
\end{gathered}
$$$$
\left(x \geq 0 ; m \in \mathbb{N}_{0} ; \alpha \in \mathbb{C} ; \beta \in \mathbb{N} ;|t|<1\right) .
$$

Proof. Similarly as in Theorem 2, we can prove the results in Theorem 5. So their details are left to the interested reader by, instead of the essential identity ((17)), presenting the following identity:

$$
\begin{gathered}
(1-\alpha-m-k)_{\beta n}=(1-\alpha-m)_{\beta n}\left(\begin{array}{c}
\alpha+m+k-1 \\
k
\end{array}\right) \\
\cdot\left(\begin{array}{c}
\alpha+m-\underset{k}{\beta n}+k-1 \\
k
\end{array}\right)^{-1}, \\
\left(k, n \in \mathbb{N}_{0} ; \beta \in \mathbb{N}\right) .
\end{gathered}
$$

It should be noted that, if we set $\beta=1$ and replace $\alpha$ by $\alpha-m$ in ((24)), we are easily led to the result ((13)).

Concluding Remarks. If we add the two generating functions ((13)) and ((14)) and use the decomposition formula ((11)), we have an interesting result expressed in terms of generalized hypergeometric functions ${ }_{p} F_{q}$ :

$$
\begin{array}{r}
\sum_{k=0}^{\infty}\left(\begin{array}{c}
\alpha+k-1 \\
k
\end{array}\right){ }_{p} F_{q+1}\left[\begin{array}{r}
\left.a_{1}, a_{2}, \ldots, a_{p} ; z\right] t^{k} \\
1-\alpha-k, b_{1}, \ldots, b_{q} ;
\end{array}\right] \\
=(1-t)^{-\alpha}{ }_{p} F_{q+1}\left[\begin{array}{r}
a_{1}, a_{2}, \ldots, a_{p} ; \\
1-\alpha, b_{1}, \ldots, b_{q} ;
\end{array}(1-t)\right], \\
(\alpha \in \mathbb{C} ;|t|<1) .
\end{array}
$$

We also observe that the result $((28))$ corresponds to that given in [28, page 170, Equation (5.12)].
The generalized incomplete hypergeometric functions given in $((7))$ and $((8))$ reduce, when $x=0$, to the generalized hypergeometric function ${ }_{p} F_{q}\left(p, q \in \mathbb{N}_{0}\right)$ whose particular cases are known to express most of the special functions occurring in the mathematical, physical, and engineering sciences. Therefore most of the known and widely investigated special functions are expressible also in terms of the generalized incomplete hypergeometric functions ${ }_{p} \gamma_{q}(p, q \in$ $\left.\mathbb{N}_{0}\right)$ and $\Gamma_{q}\left(p, q \in \mathbb{N}_{0}\right)$ (for some interesting examples and applications, see [16, Sections 5 and 6]). In view of this observation, the results presented here, being of general character, can yield numerous generating functions for a certain class of incomplete hypergeometric polynomials (see [17]) and other special functions which are expressible in terms of hypergeometric functions. Finally, we conclude our present investigation by remarking that our results presented here are also believed to give some contribution to the communication theory, probability theory, and groundwater pumping modeling.

\section{Conflict of Interests}

The authors declare that there is no conflict of interests regarding the publication of this paper.

\section{Acknowledgments}

The authors should express their deep gratitude to all the referees for their very helpful and critical comments originating from only detailed reviews of this paper by sharing their valuable time. This research was, in part, supported by the Basic Science Research Program through the National Research Foundation of Korea funded by the Ministry of Education, Science and Technology of the Republic of Korea (Grant no. 2010-0011005). This work was supported by Dongguk University Research Fund.

\section{References}

[1] F. E. Prym, "Zur theorie der gamma function," The Journal für die Reine und Angewandte Mathematik, vol. 82, pp. 165-172, 1877.

[2] M. Abramowitz and I. A. Stegun, Eds., Handbook of Mathematical Functions: with Formulas, Graphs, and Mathematical Tables, Applied Mathematics Series 55, National Bureau of Standards, Washington DC, USA, 9th edition, 1984.

[3] P. Agarwal, "Certain properties of the generalized Gauss hypergeometric functions," Applied Mathematics \& Information Sciences, vol. 8, no. 5, pp. 2315-2320, 2014.

[4] G. E. Andrews, R. Askey, and R. Roy, Special Functions, vol. 71 of Encyclopedia of Mathematics and its Applications, Cambridge University Press, Cambridge, UK, 1999.

[5] E. H. Doha and W. M. Abh-Elhameed, "New linearization formulae for the products of Chebyshev polynomials of third and fourth kind," Rocky Mountain Journal of Mathematics. In press.

[6] A. Erdélyi, W. Magnus, F. Oberhettinger, and F. G. Tricomi, Higher Transcendental Functions, vol. 1, McGraw-Hill Book Company, New York, NY, USA, 1953. 
[7] N. L. Johnson, S. Kotz, and N. Balakrishnan, Continuous Univariate Distributions, vol. 2, John Wiley \& Sons, New York, NY, USA, 1995.

[8] W. Koef, Hypergepometric Summation Vieweg, BraunschweigWiebaden, 1998.

[9] H. M. Srivastava and J. Choi, Series Associated with the Zeta and Related Functions, Kluwer Academic Publishers, Dordrecht, The Netherlands, 2001.

[10] H. M. Srivastava and J. Choi, Zeta and q-Zeta Functions and Associated Series and Integrals, Elsevier Science, Amsterdam, The Netherlands, 2012.

[11] H. M. Srivastava and P. W. Karlsson, Multiple Gaussian Hypergeometric Series, Ellis Horwood, Chichester, UK, 1985.

[12] H. M. Srivastava and B. R. K. Kashyap, Special Functions in Queuing Theory and Related Stochastic Processes, Academic Press, New York, NY, USA, 1982.

[13] N. M. Temme, Special Functions: An Introduction to Classical Functions of Mathematical Physics, John Wiley and Sons, New York, NY, USA; Brisbane and Toronto, Chichester, UK, 1996.

[14] G. N. Watson, A Treatise on the Theory of Bessel Functions, Cambridge University Press, Cambridge, UK, 2nd edition, 1944.

[15] E. T. Whittaker and G. N. Watson, A Course of Modern Analysis: An Introduction to the General Theory of Infinite Processes and of Analytic Functions; With an Account of the Principal Transcendental Functions, Cambridge University Press, New York, NY, USA, 4th edition, 1963.

[16] H. M. Srivastava, M. A. Chaudhry, and R. P. Agarwal, "The incomplete Pochhammer symbols and their applications to hypergeometric and related functions," Integral Transforms and Special Functions, vol. 23, no. 9, pp. 659-683, 2012.

[17] R. Srivastava and N. E. Cho, "Generating functions for a certain class of incomplete hypergeometric polynomials," Applied Mathematics and Computation, vol. 219, no. 6, pp. 3219-3225, 2012.

[18] F. G. Tricomi, "Sulla funzione gamma incompleta," Annali di Matematica Pura ed Applicata, vol. 31, no. 1, pp. 263-279, 1950.

[19] A. M. Legendre, Exercises de Calcul Intégral Sur Divers Orders de Transcendantes et Sur les Quadratures, vol. 1, Courcier, Paris, France, 1981.

[20] E. D. Rainville, Special Functions, Macmillan, New York, NY, USA, 1960.

[21] B. C. Carlson, Special Functions of Applied Mathematics, Academic Press, New York, NY, USA, 1977.

[22] Y. L. Luke, Mathematical Functions and Their Approximations, Academic Press, New York, NY, USA, 1975.

[23] L. J. Slater, Generalized Hypergeometric Functions, Cambridge University Press, Cambridge, UK, 1966.

[24] H. M. Srivastava and H. L. Manocha, A Treatise on Generating Functions, Halsted Press, Ellis Horwood, John Wiley \& Sons, New York, NY, USA, 1984.

[25] P. Agarwal and C. L. Koul, "On generating functions," Journal of Rajasthan Academy of Physical Sciences, vol. 2, no. 3, pp. 173180, 2003.

[26] D. Zeitlin, "A new class of generating functions for hypergeometric polynomials," Proceedings of the American Mathematical Society, vol. 25, pp. 405-412, 1970.

[27] H. M. Srivastava, "Some bilateral generating functions for a class of special functions I and II," Indagationes Mathematicae (Proceedings), vol. 83, no. 2, pp. 234-246, 1980.
[28] M.-P. Chen and H. M. Srivastava, "Orthogonality relations and generating functions for Jacobi polynomials and related hypergeometric functions," Applied Mathematics and Computation, vol. 68, no. 2-3, pp. 153-188, 1995.

[29] H. W. Gould, "A series transformation for finding convolution identities," Duke Mathematical Journal, vol. 28, pp. 193-202, 1961. 


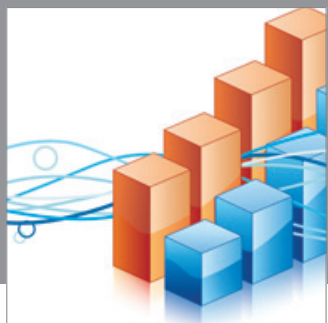

Advances in

Operations Research

mansans

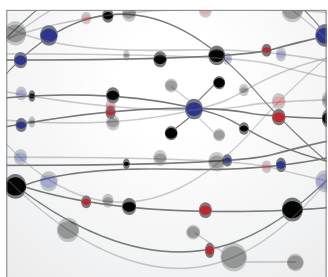

The Scientific World Journal
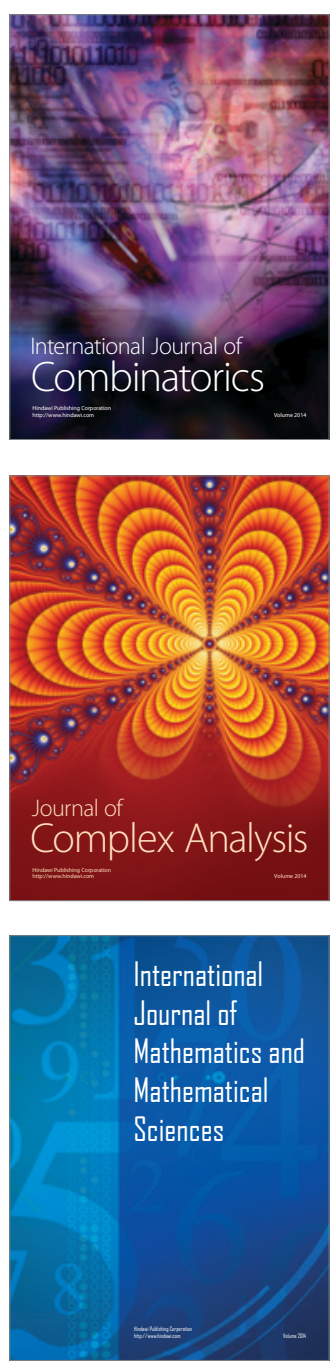
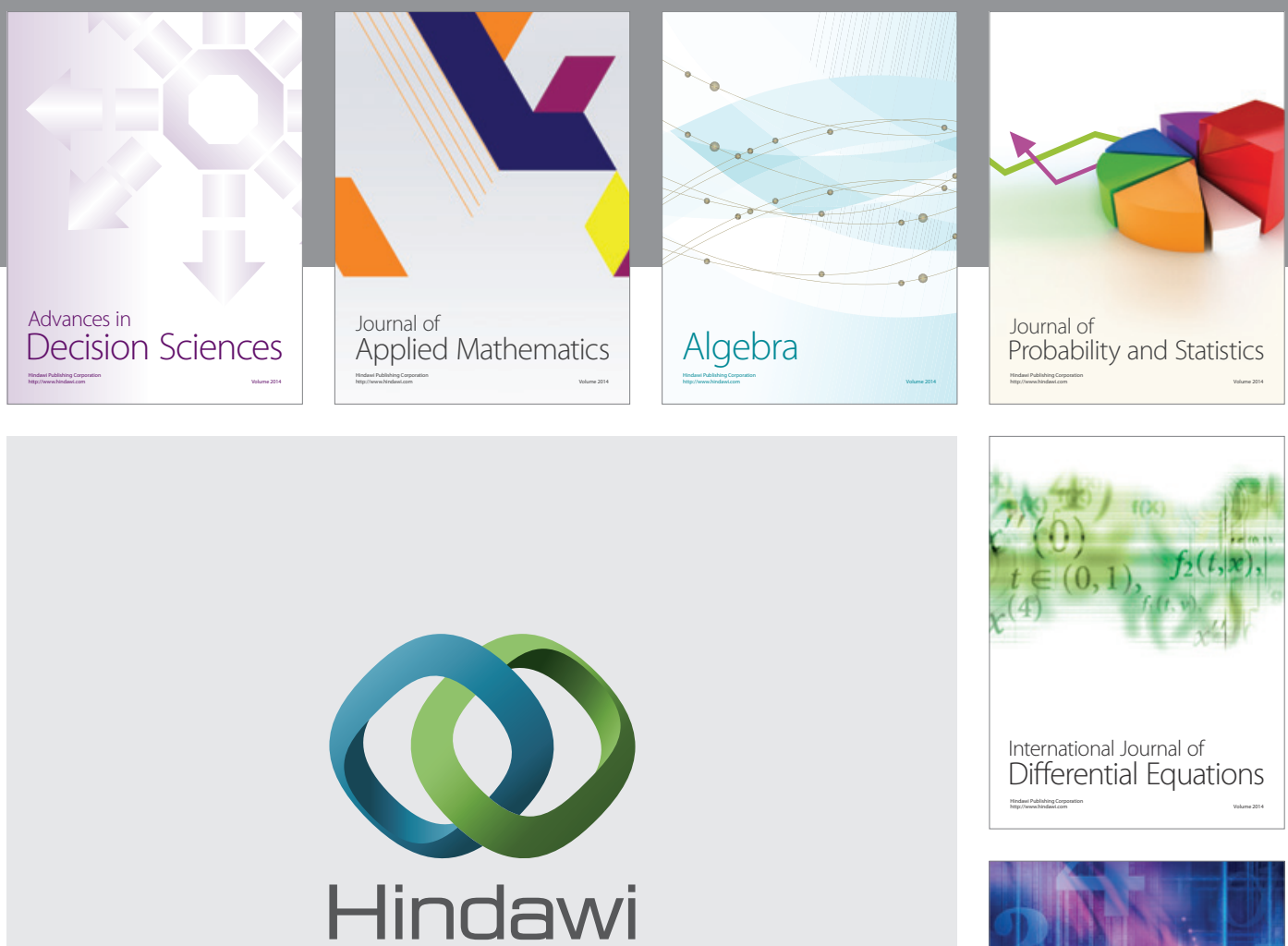

Submit your manuscripts at http://www.hindawi.com
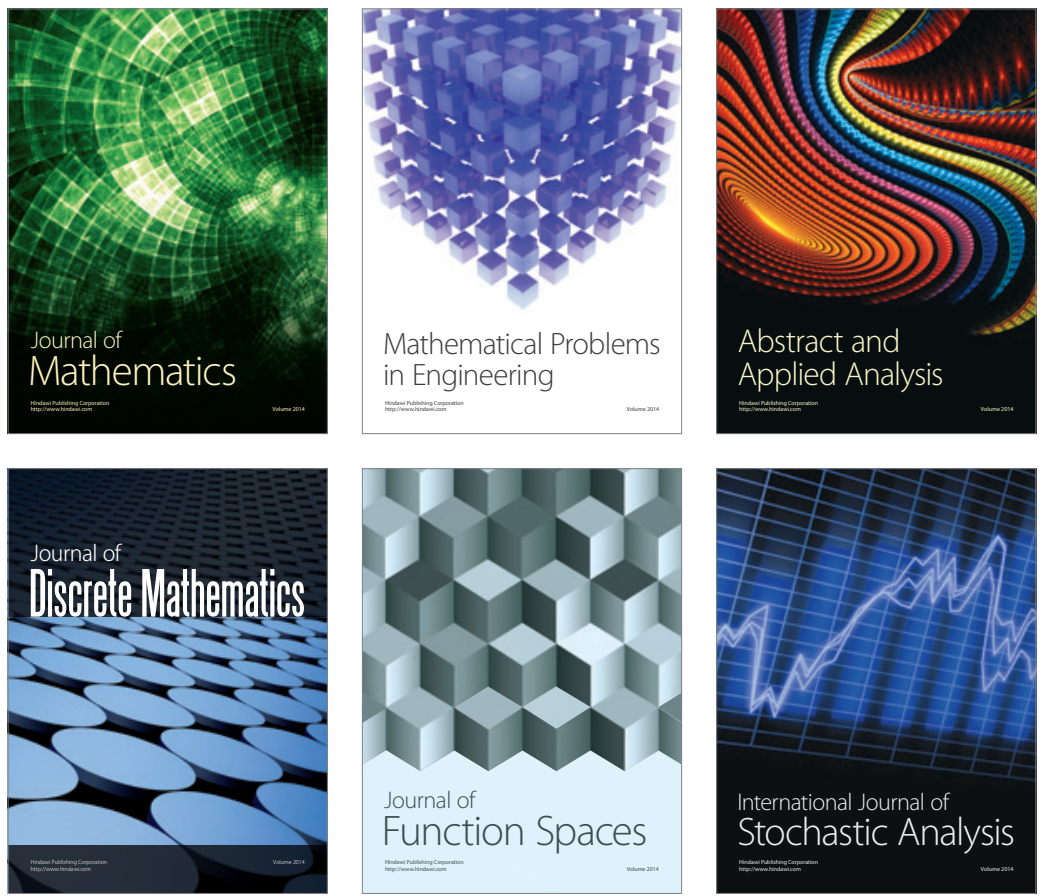

Journal of

Function Spaces

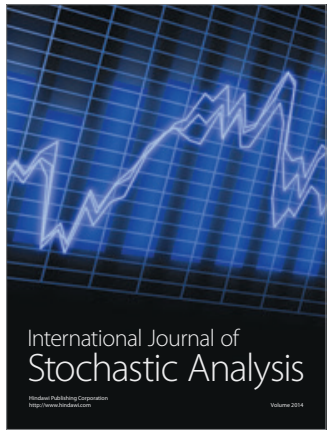

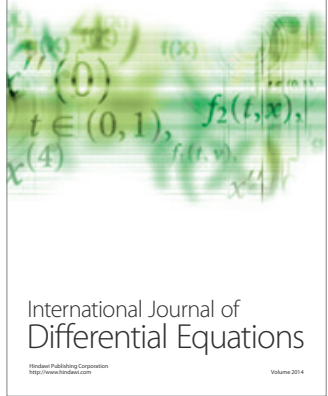
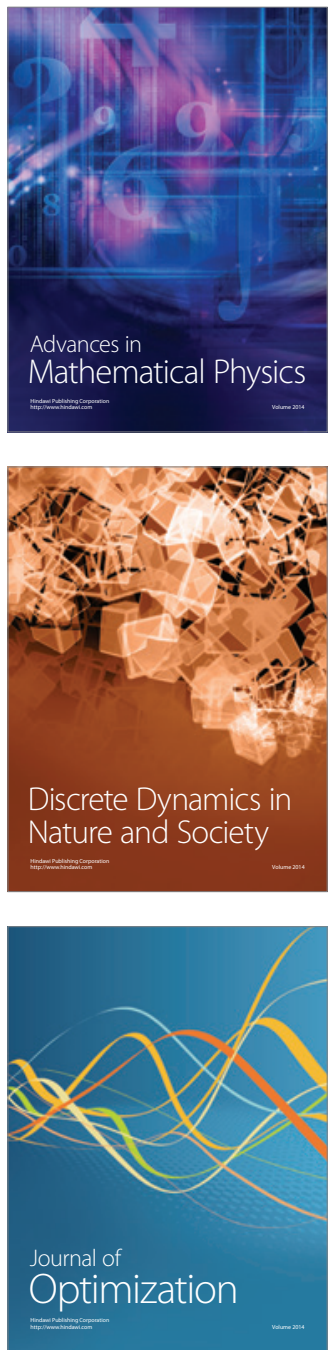